\title{
Molecular-beam epitaxy of the half-Heusler alloy NiMnSb on (In,Ga)As/InP (001)
}

\author{
P. Bach, ${ }^{\text {a) }}$ A. S. Bader, C. Rüster, C. Gould, C. R. Becker, G. Schmidt, \\ and L. W. Molenkamp \\ Physikalisches Institut (EP3), Universität Würzburg, Am Hubland, 97074 Würzburg, Germany \\ W. Weigand, C. Kumpf, and E. Umbach \\ Physikalisches Institut (EP2), Universität Würzburg, Am Hubland, 97074 Würzburg, Germany
}

R. Urban, G. Woltersdorf, and B. Heinrich

Simon Fraser University, 8888 University Drive, Burnaby, British Columbia V5A IS6, Canada

(Received 17 March 2003; accepted 4 June 2003)

\begin{abstract}
We report the growth of the half-Heusler alloy NiMnSb on InP (001) by molecular-beam epitaxy using a lattice-matched (In,Ga)As buffer. High-resolution x-ray diffraction confirms a high crystalline quality. Spot-profile analysis low-energy electron diffraction measurements show well-defined surface reconstructions. The samples show the expected high Curie temperature and an uniaxial anisotropy. (c) 2003 American Institute of Physics. [DOI: 10.1063/1.1594286]
\end{abstract}

The advancement of semiconductor spintronics is highly dependent on the availability of suitable materials. Electrical spin injection into semiconductors has been demonstrated using all-semiconductor structures. ${ }^{1,2}$ Unfortunately these spinaligners can only be used at low temperatures. It has been shown ${ }^{3}$ that ferromagnetic metals, in order to permit spin injection at room temperature in the diffusive regime without tunnel barriers, must have nearly $100 \%$ spin polarization at the Fermi level.

Half-metallic ferromagnets (HMF) meet this demand. The band structure of these materials is metallic for one spin orientation and insulating or semiconductorlike for the other. The half-Heusler alloy NiMnSb was one of the first materials for which the HMF character was predicted. ${ }^{4}$ Later experiments using positron annihilation indicated that bulk $\mathrm{NiMnSb}$ is indeed a half-metallic ferromagnet. ${ }^{5,6}$ However, experiments sensitive to the spin polarization at the surface showed a highly reduced spin polarization. ${ }^{7,8}$ It is therefore of crucial importance to have complete control on the interfaces and surfaces of the structures.

$\mathrm{NiMnSb}$ is especially well suited for applications because of its high Curie temperature $(730 \mathrm{~K})$ and the possibility to grow this material epitaxially on common semiconductors. NiMnSb crystallizes in the $\mathrm{Cl}_{b}$ structure, which consists of three interpenetrating fcc lattices at the locations A $(0,0,0), B\left(\frac{1}{4}, \frac{1}{4}, \frac{1}{4}\right), C\left(\frac{3}{4}, \frac{3}{4}, \frac{3}{4}\right)$. Site A is occupied with Ni, B with $\mathrm{Mn}$, and $\mathrm{C}$ with $\mathrm{Sb}$.

Molecular-beam epitaxy (MBE) growth of $\mathrm{NiMnSb}(001)$ layers has been investigated previously using a $\mathrm{GaAs}(001)$ substrate. ${ }^{9}$ This material system has the disadvantage of a high lattice mismatch between GaAs and NiMnSb (4.4\%), which can lead to dislocations and interface defects. The lattice constant of NiMnSb is $5.903 \AA{ }^{10}$ which implies a lattice mismatch to $\mathrm{InP}$ of only $0.6 \%$ and therefore makes this material combination an ideal candidate. In the present work we thus use InP as a substrate material. Our samples

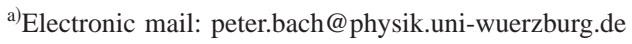

typically consist of a semi-insulating InP wafer, $200 \mathrm{~nm}$ $\left(\mathrm{In}_{0.53} \mathrm{Ga}_{0.47}\right)$ As lattice matched to InP and a NiMnSb layer of varying thickness.

The samples were grown in a multichamber MBE system, allowing transport between the different chambers under ultrahigh vacuum. Growth was monitored using reflection high-energy electron diffraction (RHEED) with an electron energy of $10 \mathrm{keV}$. The (In,Ga)As buffer was grown in a Riber 2300 system with a residual pressure of $1 \times 10^{-10}$ mbar using elemental sources with $6 \mathrm{~N}$ purity. As was evaporated with a valved cell producing $\mathrm{As}_{4}$ output, In and Ga using standard effusion cells. The NiMnSb layer was grown in a chamber built by MBE-Komponenten $\mathrm{GmbH}$ with a residual pressure of $8 \times 10^{-11}$ mbar using standard effusion cells. The purity of $\mathrm{Ni}$ was $5 \mathrm{~N}, \mathrm{Mn}$ and $\mathrm{Sb}$ were of $6 \mathrm{~N}$ purity.

All films in this work were grown on epiready, Fe doped InP wafers supplied by InPACT. The wafers were glued with In on molybdenum holders. Before entering the III- $\mathrm{V}$ chamber the substrate was heated for $10 \mathrm{~min}$ at $300^{\circ} \mathrm{C}$ in an UHV degassing station to remove any humidity from the surface. To desorb the oxide layer, the substrate temperature was raised by $20 \mathrm{~K}$ per minute until the $(2 \times 4) \mathrm{InP}$ reconstruction was observed by RHEED. The substrate was then heated for $5 \mathrm{~min}$ to a temperature $20 \mathrm{~K}$ above this point (typically $530^{\circ} \mathrm{C}$ ) and immediately cooled down $20 \mathrm{~K}$ to the growth temperature (typically $510^{\circ} \mathrm{C}$ ). During the desorption process the InP surface was stabilized above $350^{\circ} \mathrm{C}$ with an $\mathrm{As}_{4}$ overpressure. The temperatures during this growth process were measured by a Accu-Fiber pyrometer. To achieve lattice matching of the (In, Ga)As layer to InP, the flux ratio of In to $\mathrm{Ga}$ was set to 3.2. Best quality samples were achieved with a As flux $25 \times$ higher than the combined group III fluxes. The growth was initiated by simultaneous evaporation of In and $\mathrm{Ga}$. The buffer was grown for $30 \mathrm{~min}$ resulting in a thickness of typically $200 \mathrm{~nm}$. After growth the buffer was cooled down to $200^{\circ} \mathrm{C}$ under $\mathrm{As}_{4}$ flux and immediately transferred 

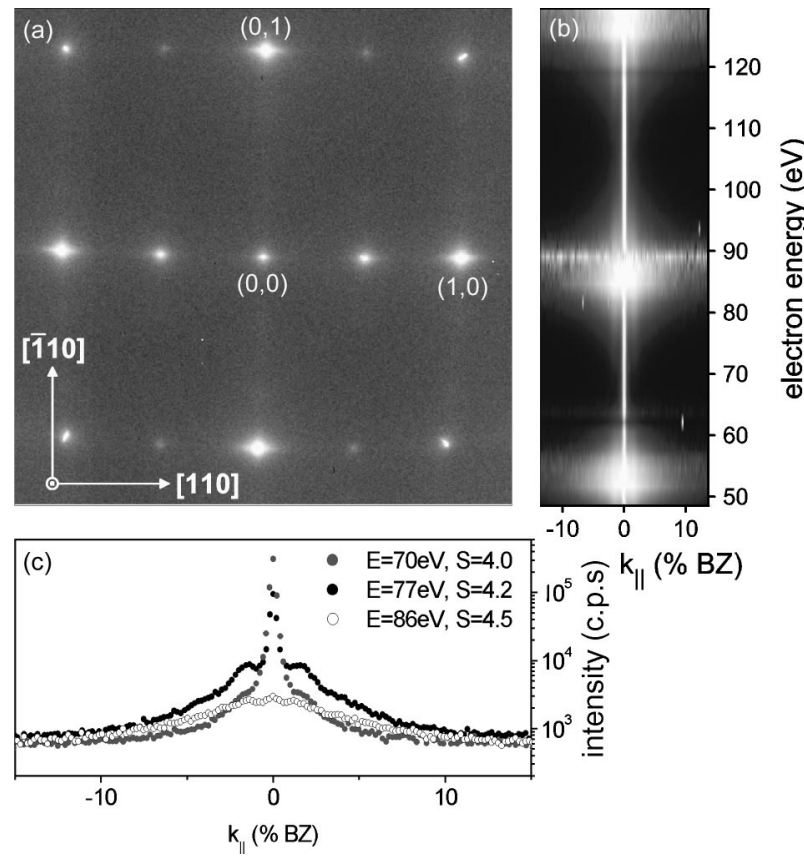

FIG. 1. SPA-LEED pattern and spot-profiles of the NiMnSb- $(2 \times 1)$ surface (a) Two-dimensional LEED pattern taken at an electron energy of 75 eV. (b) Line scans of the specular spot, taken along the [110] direction at different electron energies. (Note that each of the scans is normalized to its individual maximum.) (c) Spot profiles of the specular spot taken at different electron energies along the [110] direction.

to the NiMnSb-growth chamber under continuous UHV conditions.

$\mathrm{NiMnSb}$ was grown at a substrate temperature varying between 200 and $400{ }^{\circ} \mathrm{C}$, as measured by the thermocouple of the heating coil. Growth was started by simultaneous evaporation of $\mathrm{Ni}, \mathrm{Mn}$, and $\mathrm{Sb}$ onto the $(3 \times 4)$ reconstructed (In,Ga)As surface. The beam equivalent pressure ratios varied from 7 to 20 for $\mathrm{Sb}$ to $\mathrm{Ni}$ and 2.0 to 3.2 for $\mathrm{Mn}$ to $\mathrm{Ni}$.

Best crystalline quality was achieved using a substrate temperature of $300^{\circ} \mathrm{C}$, a $\mathrm{Mn}$ to Ni flux ratio of 2.4 and a $\mathrm{Sb}$ to Ni ratio of 14.3. After growing typically $2 \mathrm{ML}$ of $\mathrm{NiMnSb}$, a clear and streaky $(2 \times 1)$-RHEED pattern appeared. RHEED oscillations measured directly after the growth start are characteristic for a Frank-Van der Merwe layer-by-layer growth mode. The $(2 \times 1)$ reconstructed surface was examined by spot-profile analysis low-energy electron diffraction (SPA-LEED) after the growth of 10 ML NiMnSb. The diffraction pattern shows sharp diffraction spots from the $(2 \times 1)$ reconstruction [Fig. 1(a)]. Figure 1(b) shows the energy dependence of the specular spot profile. [110]-line scans were measured at different energies, background corrected, and normalized to their maximum individually. A stepinduced broadening of the specular spot is clearly visible at 53,88 , and approximately $130 \mathrm{eV}$ (just outside of the investigated energy range). These values correspond to $k$ vectors of 7.4, 9.6, and $11.7 \AA^{-1}$, respectively, and represent antiphase conditions ${ }^{11}$ for a surface with steps of a height of $2.94 \AA=a_{0} / 2$. At $k$ vectors corresponding to the $a_{0} / 4$ step height no broadening can be detected. Since for the $\mathrm{Cl}_{b}$ crystal structure $\mathrm{Ni}$ and $\mathrm{Mn} / \mathrm{Sb}$ containing layers alternate with a distance of $a_{0} / 4$ in [001] direction, the terraces are terminated by either Ni or $\mathrm{Mn}$ and $\mathrm{Sb}$. We have observed that the $(2 \times 1)$ reconstruction can be prepared from an arbitrary Downloaded 04 Jun 2005 to 132.199.212.5. Redistribution subject

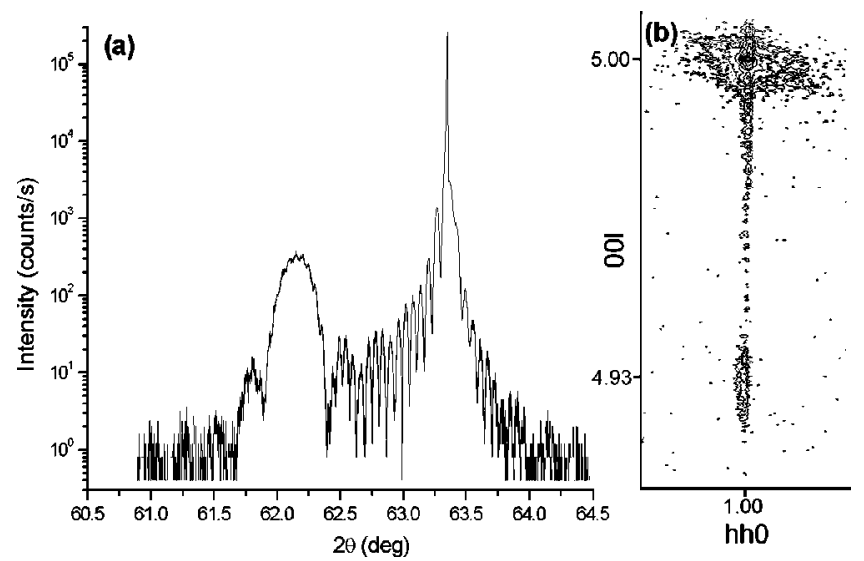

FIG. 2. (a) $\omega / 2 \theta$ scan of a $40 \mathrm{~nm} \mathrm{NiMnSb}$ layer on top of a $200 \mathrm{~nm}$ $\mathrm{In}_{0.53} \mathrm{Ga}_{0.47} \mathrm{Ga}$ buffer taken on the (004) reflection. (b) Reciprocal spacemap of the same sample taken in the vicinity of the (115) reflection.

reconstruction by a simultaneous supply of $\mathrm{Mn}$ and $\mathrm{Sb}$ atoms. This indicates that the $(2 \times 1)$ reconstructed surface is $\mathrm{Mn} / \mathrm{Sb}$ terminated. A calculation of the terrace size from the specular spot profile [Fig. 1(c)] yields an estimated average size of $150 \AA$, indicating that this surface is relatively smooth.

After growth of typically $80 \mathrm{~nm}$, the estimated critical thickness for $\mathrm{NiMnSb}$ on InP, the surface reconstruction slowly changes to a $(3 \times 3)$ reconstruction. The LEED diffraction patterns of this surface are not as clear as for the $(2 \times 1)$ reconstructed surface, but nevertheless the twodimensional LEED pattern shows clearly that this surface consists of a $(3 \times 3)$ reconstruction possibly with $180^{\circ}$ rota-
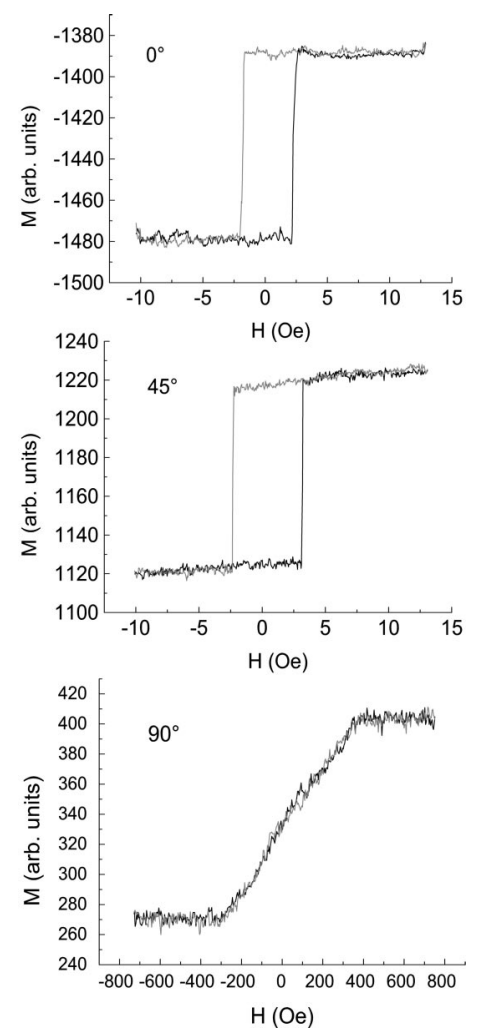

FIG. 3. Three hysteresis curves taken with different orientations of the magnetic field on a $5 \mathrm{~nm}$ thick NiMnSb layer covered with $10 \mathrm{~nm}$ of Ti. The field was rotated in-plane, an angle of $0^{\circ}$ corresponds to a field in the [110]

direction. 
tional domains. Two $(3 \times 1)$ domains, rotated by $90^{\circ}$ to each other, can be ruled out. Since this reconstruction can be prepared by exposing the surface to a $\mathrm{Ni}$ flux it can be concluded that the $(3 \times 3)$ reconstruction is terminated by $\mathrm{Ni}$ atoms.

The structural properties of the samples were characterized further by high-resolution x-ray diffraction. $\omega / 2 \theta$ scans $^{12}$ of the symmetric (004) reflection [Fig. 2(a)] show multiple thickness fringes for both the (In,Ga)As buffer and the NiMnSb layer. This indicates uniform thicknesses of both layers and a high-quality interface in between. A further indication of the excellent crystalline quality is the small full width at half maximum (FWHM) of the (004) reflection in $\omega$ direction. With a value of 12 arcsec the FWHM of the $\mathrm{NiMnSb}$ epilayer peak is exactly the value of the substrate, evidence of absence of mosaicity in this layer, within the experimental detection limit. A reciprocal spacemap of the (115) reflection [Fig. 2(b)] shows that the peaks corresponding to the substrate, buffer, thickness fringes and Heusler layer lie on a straight line in (001) direction, which proves that both buffer and NiMnSb layer are completely pseudomorphic. ${ }^{13}$

A $5 \mathrm{~nm}$ thick NiMnSb layer, covered by $10 \mathrm{~nm}$ of $\mathrm{Ti}$ in order to prevent oxidation, was characterized by magneto optical Kerr spectroscopy (Fig. 3). The hysteresis loops indicate a strong uniaxial anisotropy with the easy axis along [110]. This anisotropy was previously observed in NiMnSb layers on $\mathrm{GaAs}^{9}$ and in bulk material. ${ }^{14}$ The uniaxial anisotropy thus appears to be a bulk property. We have, however, observed that an additional fourfold anisotropy occurs in layers of thicknesses around $20 \mathrm{~nm}$. This will be discussed in more detail in a future publication. Temperature dependent superconducting quantum interference device measurements show a drop in the magnetization of only $5 \%$ from 2 to 400 $\mathrm{K}$. This is consistent with a Curie temperature of bulk $\mathrm{NiMnSb}$ of $730 \mathrm{~K}$.

In conclusion we have investigated the growth of NiMnSb on InP (001). The obtained samples show a high crystalline quality and well-defined surface reconstructions. A study of the magnetic properties revealed the expected high Curie temperature and a strong uniaxial anisotropy.

The authors acknowledge funding by the German BMBF, the European Commission, and the German Science Foundation through SFB 410.

${ }^{1}$ R. Fiederling, G. Reuscher, W. Ossau, G. Schmidt, A. Waag, and L. W. Molenkamp, Nature (London) 402, 787 (2000).

${ }^{2}$ Y. Ohno, D. K. Young, B. F. Matsukara, H. Ohno, and D. D. Awschalom, Nature (London) 402, 790 (2000).

${ }^{3}$ G. Schmidt, L. Molenkamp, A. T. Filip, and B. J. van Wees, Phys. Rev. B 62, R4790 (2000).

${ }^{4}$ R. de Groot, F. Mueller, P. van Engen, and K. Buschow, Phys. Rev. Lett. 50, 2024 (1983).

${ }^{5}$ K. Hanssen and P. Mijnarend, Phys. Rev. B 34, 5009 (1986).

${ }^{6}$ K. Hanssen, P. Mijnarends, L. Rabou, and K. Buschow, Phys. Rev. B 42, 1533 (1990)

${ }^{7}$ R. Soulen, Jr., J. Byers, M. Osofsky, B. Nadgorny, T. Ambrose, S. Cheng, P. Broussard, C. Tanaka, J. Nowak, J. Moodera, A. Barry, and J. Coey, Science 282, 85 (1998).

${ }^{8}$ D. Ristoiu, J. Nozieres, C. Borca, T. Komesu, H. Jeong, and P. Dowben, Europhys. Lett. 49, 624 (2000).

${ }^{9}$ W. Van Roy, J. D. Boeck, B. Brijs, and G. Borghs, Appl. Phys. Lett. 77, 4190 (2000).

${ }^{10}$ L. Castelliz, Z. Metallkd. 46, 201 (1955).

${ }^{11}$ M. Horn von Hoegen, Z. Kristallogr. 214, 684 (1999).

${ }^{12}$ G. Bauer and W. Richter, Optical Characterisation of Epitaxial Semiconductor Layers, 1. Auflage (Springer, Berlin, 1996).

${ }^{13}$ V. Holý, U. Pietsch, and T. Baumbach, High Resolution X-Ray Scattering from Thin Films and Multilayers (Springer, Berlin, 1999).

${ }^{14}$ C. Hordequin, J. Pierre, and R. Currat, J. Magn. Magn. Mater. 162, 75 (1996). 